\title{
Lifetimes of selective-adsorption resonances in atom-surface elastic scattering
}

\author{
Marta I. Hernández, José Campos-Martínez, and S. Miret-Artés \\ Instituto de Matemáticas y Física Fundamental Consejo Superior de Investigáciones Científicas, \\ Serrano 123, 28006 Madrid, Spain \\ Rob D. Coalson \\ Department of Chemistry, University of Pittsburgh, Pittsburgh, Pennsylvania 15260
}

(Received 1 October 1993)

\begin{abstract}
From a time-dependent calculation and by assuming an exponential decay law for the resonances formed, we have computed lifetimes of selective-adsorption resonances (SAR's) in the elastic scattering of $\mathrm{He}$ atoms from the $\mathrm{Cu}(110)$ and $\mathrm{Cu}(117)$ surfaces. In previous works, where SAR's were studied within the time-independent close-coupling method, we found that in addition to the energy width obtained by analyzing plots of diffraction intensities versus the energy of the incident beam, another internal energy width (with a very different numerical value) can be defined. Here we show that it is internal width that can be directly related to the lifetime of the resonance. Also, we have performed a simulation of the half collision or "selective-desorption" process. This approach is shown to yield accurate values for lifetimes of SAR's.
\end{abstract}

\section{INTRODUCTION}

Atom-surface scattering is widely recognized as a very useful tool in the study of solid surface properties. In particular, the scattering of $\mathrm{He}$ beams from various surfaces, both insulator and metallic in nature, has attracted the attention of a considerable group of theoreticians and experimentalists. ${ }^{1}$ Among processes that take place during atom-surface collisions, selective adsorption resonances (SAR's) allow an accurate way of determining bound-state energies associated with the atom-surface interaction potential, and hence provide a unique way to build precise interaction potentials as well as an important test for any theoretical method. ${ }^{2}$ Moreover, it has recently been shown that selective adsorption and selective desorption as well play important roles as steps in more complex processes involving trapping or sticking. ${ }^{3}$

In previous works on SAR's, we have shown by means of the time-independent close-coupling (TICC) method ${ }^{4}$ that there is a "hierarchy" in the variables controlling the atom-surface scattering process. First, there are the experimentally controllable variables: the energy $E$ and the polar and azimuthal angles $(\theta$ and $\phi)$ of the incident beam. Second, there is a set of "internal variables" which are the kinetic-energy components of the different diffraction channels along the perpendicular direction to the surface. A SAR is observed as a sharp change in the diffraction intensities, usually in the form of a Lorentzian profile, when they are plotted versus $E$, $\theta$, or $\phi$. In this way, different widths of the resonance, $\Gamma_{E}, \Gamma_{\theta}$, and $\Gamma_{\phi}$, can be determined (as the width at half height in a fit to a Lorentzian function) by scanning the energy, polar or azimuthal angles, respectively, of the incident beam. On the other hand, an internal width of the resonance $\Gamma_{\text {int }}$ can be also defined through the changes experienced by one of the internal variables, specifically, the perpendicular kinetic-energy component associated with the resonant channel. The question then arises as to which width directly provides an estimation of the lifetime $\tau$ of the resonant state via the standard relationship $\tau=\hbar / \Gamma$. In the present case one might have difficulty in assigning the $\Gamma$, whose inverse correlates with a measurement of the lifetime, since there are two widths with energy units. In fact, they can differ by up to one or two orders of magnitude. ${ }^{4}$ Estimations of lifetimes would be very valuable since longer or shorter residence times of the atom traveling on the surface could enhance the probability of appearance of other processes, for instance, the interaction with phonons and defects.

In order to resolve the question just posed, we have studied such resonances in a completely different way, by means of wave-packet propagation on a grid, where no concept of "channels" or "internal variables" is introduced and where all the physical magnitudes are extracted from the interaction of a wave packet with the full atom-surface potential. Time-dependent wave-packet propagation has become a very important technique for treating the quantum dynamics of large molecular systems. At present, these methods are often more efficient than more traditional time-independent methods when studying very fast processes (for instance, in the case of photodissociation, where typical propagation times are subpicosecond). Application of these methods to atomsurface scattering has also been successful, mainly due to the adequacy of the use of Cartesian coordinates, which are the most appropriate coordinates for describing this scattering process and, at the same time, are the best for employing one of the most popular methods in evaluating the kinetic-energy terms within the Hamiltonian, the fast Fourier transform (FFT) algorithm. ${ }^{5}$ Indeed, one of the pioneering works using the FFT by Yinonn and Kosloff ${ }^{6}$ dealt precisely with atom-surface scattering. Timedependent methods have since been used to treat atomsurface elastic and inelastic scattering, scattering when defects and adsorbates are present on the surface, and many other related processes. ${ }^{7}$ However, time-dependent 
methods have been used less extensively when resonances are present in the scattering of atoms and molecules from surfaces, mainly because of problems resulting from the large increase in propagation time needed due to the formation of a quasistationary state. Important exceptions have been the works by Kosloff and co-workers ${ }^{6,8}$ and Mowrey and Kouri. ${ }^{9}$

In this work two approaches to the problem have been employed. First, we have studied the full collision process and monitored the decay rate of the adsorbed wave packet. Second, the half-collision analog, i.e., selective desorption, has been simulated and a similar procedure to the one used in the full collision case has been carried out in order to obtain the lifetime of the desorbing wave packet.

The paper is organized as follows. In Sec. II the problem of selective-adsorption resonances is set up through the close-coupling formalism, then the wave-packet procedure to compute lifetimes is discussed, and the potential-energy surface used in all the calculations is described. Section III presents the results obtained, and discussion is presented in Sec. IV, and concluding remarks are in Sec. V.

\section{THEORETICAL BACKGROUND}

\section{A. Statement of the problem}

Consider the elastic scattering of a gas atom of mass $\mu$ and incident wave vector $\mathbf{k}$ from a static, corrugated periodic surface (a more detailed exposition can be found in Ref. 4). It is common to characterize the incidence of the atom by the set of parameters $(E, \theta, \phi)$, where $E$ is the total energy and $\theta$ and $\phi$ are the polar and azimuthal angles, respectively. Due to the periodicity of the surface, the interaction potential $V(\mathbf{r})$ is usually expanded in a Fourier series. Moreover, the behavior of the stationary wave function is given by Bloch's theorem:

$$
\Psi(\mathbf{r})=\sum_{\mathbf{G}} \psi_{\mathbf{G}}(z) \exp [i(\mathbf{K}+\mathbf{G}) \cdot \mathbf{R}],
$$

where $\mathbf{r}=(\mathbf{R}, z), z$ represents the outward normal direction to the surface plane, $\mathbf{R}=(x, y)$ describes motion along the surface, $\mathbf{K}=\left(2 \mu E / \hbar^{2}\right)^{1 / 2} \sin \theta(\cos \phi, \sin \phi)$ is the parallel component of $\mathbf{k}$, and $\mathbf{G}$ is a reciprocal lattice vector, which for the simplest case of rectangular lattices with sides $a$ and $b$ can be written as $\mathbf{G}=(2 \pi l / a, 2 \pi j / b)$ with $l, j$ integers. Starting from the stationary Schrödinger equation and integrating over the unit cell leads to the following set of close-coupling equations:

$$
\begin{aligned}
& {\left[\frac{\hbar^{2}}{2 \mu} \frac{d^{2}}{d z^{2}}+\mathscr{E}_{\mathrm{G}}(E, \theta, \phi)-V_{0}(z)\right] \psi_{\mathrm{G}}(z)} \\
& =\sum_{\mathbf{G}^{\prime} \neq \mathbf{G}} V_{\mathrm{G}-\mathrm{G}^{\prime}}(z) \psi_{\mathrm{G}^{\prime}}(z),
\end{aligned}
$$

where the functions $V_{\mathbf{G}}(z)$ are the Fourier coefficients of the interaction potential $V(r)$. The term $\mathscr{E}_{G}$ is the kinetic-energy component (along the perpendicular direction to the surface) of a diffraction channel labeled by $\mathbf{G}$ :

$$
\mathscr{E}_{\mathbf{G}}(E, \theta, \phi)=E-\frac{\hbar^{2}}{2 \mu}|\mathbf{K}+\mathbf{G}|^{2} .
$$

The $\mathscr{E}_{G}$ 's are what we have called "internal variables." They appear as independent variables in the boundary conditions for the wave function, through which the scattering matrix $\mathcal{S}$ is defined. ${ }^{10}$ Diffraction intensities are given by the square modulus of the $\mathcal{S}$-matrix elements, $\left|\mathcal{S}_{G 0}\right|^{2}$. In this way, dependence of diffraction intensities on $(E, \theta, \phi)$ is provided through this set of internal variables.

Some further remarks are worthwhile in order to understand selective-adsorption resonances. It is we known that this phenomenon occurs when the kinetic energy of a given closed channel $\rho$ (the resonant channel) is equal to an energy level $\varepsilon_{\alpha}$ of the laterally averaged potential $V_{0}(z)$ :

$$
\mathscr{E}_{\rho}(\bar{E}, \bar{\theta}, \bar{\phi})=\varepsilon_{\alpha}<0
$$

The adsorption process can be pictured as a transfer of momentum from the perpendicular to the parallel direction of the surface, resulting in a quasibound state in the perpendicular direction, while the atom is moving faster along the parallel direction. These resonances are usually labeled by $\left(\begin{array}{c}m n \\ \alpha\end{array}\right)$, where $m$ and $n$ are numbers characterizing the resonant diffraction channel by means of the reciprocal lattice vector indexes $l=m, j=n$.

In this scheme, we define $\varepsilon_{\alpha}$ as the "internal" position for the resonance because it is related to what we have called the internal variable $\mathscr{E}_{\rho}$. To this internal position there is associated an internal width $\Gamma_{\text {int }}$, the energy region where sharp spikes in the shape of the $\mathcal{S}$ matrix can be observed as $\mathscr{E}_{\rho}$ changes. ${ }^{4}$ Notice that because $\Gamma_{\text {int }}$ is defined through variations in one of the internal variables $\left(\mathscr{E}_{\rho}\right)$ it is not experimentally accessible, and therefore designation of this width as internal seems natural.

As concerns the experimentally accessible, external variables $(E, \theta, \phi)$, the "external position" of the resonance is denoted by $(\bar{E}, \bar{\theta}, \bar{\phi})$ in Eq. (4). However, it should be noticed that in Eq. (4) there are infinite combinations of the external parameters fulfilling such an equation. For different combinations of $(\bar{E}, \bar{\theta}, \bar{\phi})$, features of a given $\left(\begin{array}{c}m n \\ \alpha\end{array}\right)$ resonance (for instance, internal widths) can change drastically. For this reason, in the results section we will specify not only the label $\left(\begin{array}{c}m n \\ \alpha\end{array}\right)$ of a resonance, but also the specific values of $(\bar{E}, \bar{\theta}, \bar{\phi})$ at which we are studying such a resonance.

Also, external widths can be associated with each of the three incidence parameters $(E, \theta, \phi)$. For instance, the external energy width $\Gamma_{E}$ can be obtained analyzing plots of diffraction intensities as a function of $E$, while the incidence angles are held at the resonant position $(\bar{\theta}, \bar{\phi})$. These quantities have been shown to be related to $\Gamma_{\text {int }}$ through the following expression: ${ }^{4}$

$\Gamma_{\mathrm{int}}=\left|\mathscr{E}_{\rho}\left(\bar{E}+\Gamma_{E} / 2, \bar{\theta}, \bar{\phi}\right)-\mathscr{E}_{\rho}\left(\bar{E}-\Gamma_{E} / 2, \bar{\theta}, \bar{\phi}\right)\right|$.

This equation (as well as similar ones for the external angular widths) can be used in two ways. $\Gamma_{\text {int }}$ can be obtained when $\Gamma_{E}$ is already known by analyzing the plots of the diffraction intensities as functions of $E$ (by fitting to 
a Lorentzian shape, for instance). Or, conversely, the external widths (the ones which can be directly compared with experimental results) can be obtained from the internal width once the latter has been determined by a theoretical method.

We have just seen that two widths with energy dimensions can be defined. In order to see whether $\Gamma_{E}$ or $\Gamma_{\text {int }}$ fulfills the typical inverse relationship with lifetimes, it proves fruitful to study this problem from a completely different point of view, i.e., time-dependent methods via which lifetimes can be directly computed.

\section{B. Wave-packet propagation}

Once the problem has been briefly sketched from a time-dependent point of view, the way to proceed entails the propagation of an initial wave packet according to the appropriate time-dependent Schrödinger equation

$$
i \hbar \frac{\partial}{\partial t} \Psi(\mathbf{r}, t)=H \Psi(\mathbf{r}, t) \text {. }
$$

Assuming a plane wave as the incident wave packet, the corresponding diffraction intensities can be calculated as the square modulus of the $\mathcal{S}$-matrix elements, obtained from the usual asymptotic projection of the wave packet (when it is far away from the surface), onto diffracted states (represented by outgoing plane waves): ${ }^{11}$

$$
\begin{aligned}
\mathcal{S}\left(\mathbf{k}^{\prime} \leftarrow \mathbf{k}\right)= & \left(-k_{z} / k_{z}^{\prime}\right)^{1 / 2} N \\
& \times \int d \mathbf{r} \exp \left(-i \mathbf{k}^{\prime} \cdot \mathbf{r}\right) \Psi_{\text {out }}(\mathbf{r}, t \rightarrow \infty) .
\end{aligned}
$$

Here $\mathbf{k}, \mathbf{k}^{\prime}$ are the initial and final wave vectors, respectively, $\left(-k_{z} / k_{z}^{\prime}\right)^{1 / 2}$ is the flux conserving prefactor, $N$ contains normalization factors, and $\Psi_{\text {out }}(\mathbf{r}, t \rightarrow \infty)$ is, in practice, our wave packet once it has been propagated for a time long enough that the interaction potential can be assumed to be negligible. Notice that in this expression the Bragg condition has not been imposed.

One straightforward way to obtain external widths would involve computing $\mathcal{S}$-matrix elements at different energies (or angles) and analyzing the shape of the diffraction intensities. Provided that the wave-packet propagation is accurate, we would obtain, of course, exactly the same result as with a time-independent formalism. Such a procedure would involve extensive computational effort, since many propagations at several energies or angles must be carried out. In this work, however, our main goal is to directly study the lifetime of the quasistationary state formed, or, in other words, to watch the quasistationary state and its evolution with time. If we focus on the state formed during the course of the collision, we can characterize the decay of this state by monitoring the "restricted norm" of the wave packet in a particular spatial region $\mathcal{R}$ with time:

$$
P_{\mathcal{R}}(t)=\int_{\mathscr{R}}|\Psi(\mathbf{r}, t)|^{2} d \mathbf{r} .
$$

From the plot of this quantity versus time we can fit an exponential function and hence obtain a value for the lifetime. In this case no assumptions are made about any internal or external width. Now we only need to propagate our initial wave packet once, making sure that it fulfills the resonant condition with respect to the total energy $\bar{E}$ and angle $\bar{\theta}$. In this case, we cannot say that the value so obtained corresponds experimentally to a change in energy of the incident beam or in the incident angle. From the numerical comparison with our previous (TICC) results we will be able to see whether the lifetime corresponds to the internal width or the external width.

Evaluation of lifetimes by looking directly at the exponential decay of a restricted norm has been done previously in molecular studies ${ }^{12}$ as well as in atom-surface scattering, ${ }^{6,8}$ although the aspects investigated here have not been considered before.

In order to perform the simulation of the scattering process, calculations have been carried out starting with an initial wave packet placed very far away from the surface and using the fact that a plane wave can be written as $^{11}$

$$
\begin{aligned}
& \Psi(\mathbf{r}, 0)= \frac{1}{\sqrt{k_{z}}} \exp [i \mathbf{k} \cdot \mathbf{r}] \\
&=C \int \exp \left[\frac{i}{\hbar}\left(\mathbf{r}-\mathbf{r}_{0}\right) \cdot A_{0} \cdot\left(\mathbf{r}-\mathbf{r}_{0}\right)\right. \\
& \\
&\left.\quad+i \mathbf{k} \cdot\left(\mathbf{r}-\mathbf{r}_{0}\right)+i \mathbf{k} \cdot \mathbf{r}_{0}\right] d \mathbf{r}_{0} .
\end{aligned}
$$

In Eq. (9), the integrand is a Gaussian wave packet, with $A_{0}$ a complex matrix (having the same dimensionality as r) that gives the spread of the Gaussian function and the correlation among the components. By breaking up the integral into a discrete sum of Gaussian functions one can obtain an approximation to a plane wave over a large enough region (covering a number of units cells in the parallel direction). As more Gaussian functions are added up and a more extended region is considered, a more "monoenergetic" wave packet is built. This will turn out to be important as discussed below. The initial wave packet is then propagated in time until, after diffraction, it reaches the asymptotic region again. Once the propagation has been carried out, diffraction intensities can be computed by using Eq. (7). This can be useful in order to check the accuracy of the calculations by comparing with time-independent results. Finally, we will fit $P_{\mathcal{R}}(t)$ [Eq. (8)] to an exponential in order to get lifetimes.

We have also carried out the simulation of the selective desorption process, suggested originally by LennardJones and Devonshire. ${ }^{13}$ This would be the inverse of selective adsorption, that is, transfer of momentum from the parallel to the perpendicular direction. The purpose here is to investigate if the desorption rate of a given metastable state can provide a good approximation to the lifetime of the resonance. From our previous work ${ }^{14}$ we know that the time-independent golden rule is a useful approximation, i.e., a diabatic decoupling scheme works quite well in the systems under study. We have thus chosen as the initial wave packet a simple direct product of two one-dimensional functions:

$$
\Psi(\mathbf{r}, t=0)=\psi_{\alpha}(z) \exp [i(\mathbf{K}+\boldsymbol{\rho}) \cdot \mathbf{R}],
$$

where $\psi_{\alpha}(z)$ is an eigenstate of Eq. (2) when the inter- 


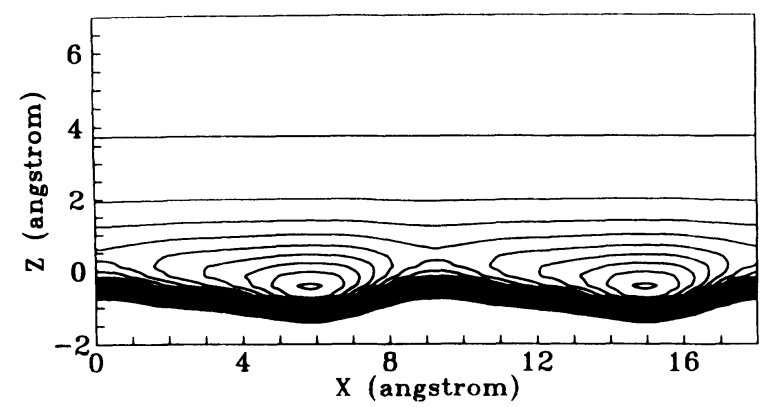

FIG. 1. Contour plot of the potential energy surface for the $\mathrm{He} / \mathrm{Cu}(117)$ system. The distance between equipotential lines is $1.25 \mathrm{meV}$; the two minima observed correspond to -10.25 $\mathrm{meV}$.

channel couplings are set to zero (diabatic approach) and the other factor is a diffracted plane wave corresponding to the resonant reciprocal lattice vector $\rho$. This plane wave can be modeled as a suitable sum of Gaussian wave packets (as described above), or, taking full advantage of the periodicity of the surface, an extended wave packet subject to periodic boundary conditions can be used instead (cf. below). This initial wave packet is also propagated according to the Schrödinger equation and, analogously to the case discussed above, the lifetime is computed using Eq. (8).

\section{The interaction potential}

The surfaces $\mathrm{Cu}(110)$ and $\mathrm{Cu}(117)$ are characterized by very weak, essentially negligible, corrugation along one of the surface axes (say the $y$ axis, in a framework where $x$ is the coordinate that describes the other parallel direction to the surface and $z$ the perpendicular one). These surfaces are ideal for theoretical purposes because of the reduction in the number of degrees of freedom involved. In particular, by considering an essentially twodimensional problem, ${ }^{15}$ theoretical results comparable with the experimental ones can be easily obtained. The potential used here was a "corrugated Morse" potential, which comes from fitting experimental results ${ }^{15}$ at 21 $\mathrm{meV}$. It is given explicitly by

$$
V(x, z)=D\left[e^{-2 x[z-\zeta(x)]}-2 e^{-\chi z}\right],
$$

where $\zeta(x)$ is the so-called "corrugation function" which reproduces the periodicity of the surface. As an illustration, a contour plot of the $\mathrm{He} / \mathrm{Cu}(117)$ potential is displayed in Fig. 1. The potential parameters are those of Ref. 15 and are the same ones previously used in our TICC calculations. ${ }^{4,14}$

\section{RESULTS}

With the potential described in Sec. II C, we have propagated all our initial wave packets, discretized on a grid, using the Feit-Fleck split operator propagator ${ }^{16}$ and the fast Fourier transform. Special care has been taken in order to accommodate maximum $k$ values on the grid, i.e., $k_{x} \ll 2 \pi / d x, k_{z} \ll 2 \pi / d z$. The two cases studied,
$\mathrm{He} / \mathrm{Cu}(110)$ and $\mathrm{He} / \mathrm{Cu}(117)$, represent examples of systems with small and large corrugation, respectively. We will present results of the full collision (selective adsorption) and half collision (selective desorption) separately.

\section{A. Resonant scattering process}

In order to check the accuracy of our wave-packet propagations, we have first computed $\mathcal{S}$-matrix elements in resonant conditions and compared with TICC results. The main problem in doing this arises from the fact that for a long-lived resonance, a significant part of the wave packet remains attached to (adsorbed on) the surface for a long time. We must propagate for a very long time in order to allow the whole wave packet to escape from the surface into the asymptotic region where Eq. (7) can be used. For instance, in the case of the $\mathrm{He} / \mathrm{Cu}(110)$ system, stretching our computational resources, a portion of the wave packet still remained on the surface and therefore the asymptotic projection could not be applied. For the case of $\mathrm{He} / \mathrm{Cu}(117)$, however, where shorter lifetimes are expected, we have observed that the leakage of the adsorbed wave packet occurs on approximately the same time scale as the direct scattering. Hence, after a prudently long time, the adsorbed wave packet can be considered negligible and Eq. (7) applied. In this case we expect the diffraction intensities obtained to be in good agreement with those computed by the TICC method. This is shown in Fig. 2, where time-dependent results (full line) are compared with TICC ones (triangles) for the resonance $\left(\begin{array}{c}50 \\ 1\end{array}\right)$ at the same resonant position, $\bar{E}=16 \mathrm{meV}$ and $\bar{\theta}=25.8^{\circ}$. As an illustration of how accurate diffraction intensities computed with this method can be, stars mark another TICC calculation at slightly nonresonant incident conditions, specifically for $\theta=22^{\circ}$. The accord is highlighted by pointing out the difference between these results and TICC values corresponding to resonant conditions. Note that in this time-dependent calculation the Bragg condition is not imposed, as is done in a TICC computation where the surface is assumed to

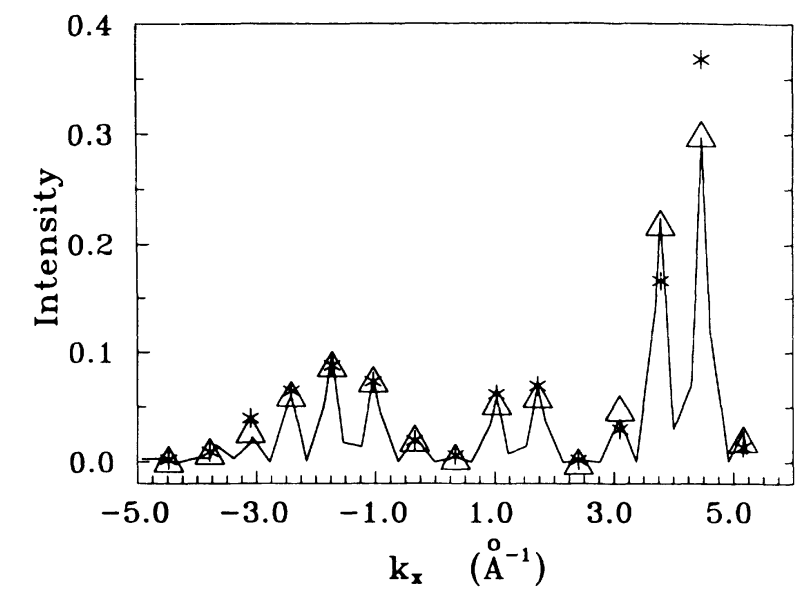

FIG. 2. Diffraction intensities as a function of $k_{x}$. The full line is the time-dependent result. The triangles are TICC results under resonant conditions. The stars are TICC results at an angle slightly out of resonance (see text). 
be infinitely periodic. However, it is easily seen that we reproduce the Bragg peaks, albeit not infinitely narrow, as discussed by Drolshagen and Heller. ${ }^{11}$ We have checked that as we increase the extension of the initial wave packet to cover more and more surface unit cells, these peaks become narrower because the interference in the interpeak region becomes more and more destructive.

Turning to the computation of lifetimes, a complementary situation was found for the surface with large corrugation, $\mathrm{He} / \mathrm{Cu}(117)$, as compared with the less corrugated $\mathrm{He} / \mathrm{Cu}(110)$. That is, on one hand, lifetimes are expected to be very long for the $\mathrm{He} / \mathrm{Cu}(110)$ system and thus diffraction intensities are difficult to compute. For $\mathrm{He} / \mathrm{Cu}(117)$, by contrast, it was easy to obtain very good results for diffraction intensities. However, it turned out to be very difficult to obtain a good fit of the decaying of the restricted norm with time, because for this system lifetimes are so short that the direct scattering of the wave packet is inextricably mixed with the leakage of the adsorbed part. Ultimately, the fitting could only be satisfactory done for $\mathrm{He} / \mathrm{Cu}(110)$.

Specifically, we have performed calculations at $\bar{E}=13$ $\mathrm{meV}$ and $\bar{\theta}=54.8992^{\circ}$ for the system $\mathrm{He} / \mathrm{Cu}(110)$ and the resonance $\left(\begin{array}{c}1 \\ 0 \\ 0\end{array}\right)$. The parameters used in the propagation are given in Table I, and the initial wave packet was an approximation to a plane wave given by Eq. (9). The behavior of the "restricted norm" $P_{\mathcal{R}}(t)$ is shown in Fig. 3. The region $\mathcal{R}$ has been taken to extend along the entire grid parallel to the surface and out to $10 \AA$ in the direction perpendicular to the surface. In the beginning, $t=0, P_{\mathcal{R}}$ is zero since the wave packet has not entered the region $\mathcal{R}$ yet. As the wave packet evolves in time, this magnitude becomes larger, reaching a value of one, and hence indicating that the wave packet is completely inside $R$. Finally, the process of desorption can be observed. The nonresonant part of the wave packet is immediately scattered back from the surface, whereas a small but significant part of it remains adsorbed. This fact is reflected in Fig. 3 by the portion of $P_{\mathcal{R}}(t)$ that appears to run almost parallel to the time axis. Although it is difficult to discern on the scale of resolution of the plot, that line represents the slow leakage of the adsorbed wave packet and in fact can be fit to an exponential function, yielding the results shown in Table II. For the sake of comparison we have added the "associated width" $\Gamma(T D)$, extracted as the inverse of the lifetime together with the TICC results concerning internal and external widths. We see that this magnitude corresponds unambiguously to the internal width $\Gamma_{\text {int }}(C C)$. Notice that if the lifetime were the inverse of the external width, its

TABLE I. Grid parameters used for studying the scattering of $\mathrm{He}$ from $\mathrm{Cu}(110)$.

\begin{tabular}{cc}
\hline \hline$E(\mathrm{meV})$ & 13.00 \\
$n x$ & 1024 \\
$n z$ & 512 \\
$d x(\AA)$ & 0.01 \\
$d z(\AA)$ & 0.01 \\
\hline \hline
\end{tabular}

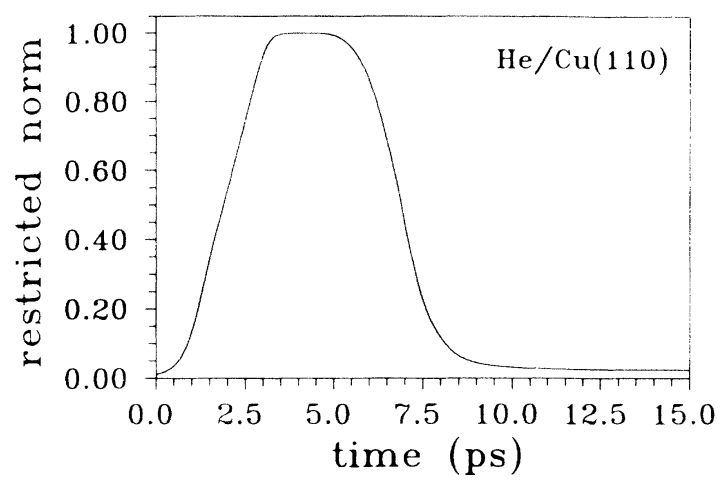

FIG. 3. Restricted norm in the scattering of $\mathrm{He}$ from $\mathrm{Cu}(110)$.

value would be as short as $6.11 \mathrm{ps}$. The evolution with time of the traveling wave-packet probability densities is shown in Fig. 4. All the features described above and illustrated in Fig. 3 are emphasized in Fig. 4 from a direct temporal perspective. In particular, a portion of the adsorbed wave packet can be seen traveling parallel to the surface at the bottom of the panel.

\section{B. Simulation of the selective-desorption process}

If in the course of the collision event a quasistationary complex is formed, it is intuitively appealing to divide the process into two components, namely, direct scattering and the decay of the resonant complex. In order to get insight into the second process and to find out if this simpler process (as compared with the full collision) can provide a good approximation to the lifetime, we have performed propagations in time of the initial wave packet given by Eq. (10).

One advantage of this approach is the reduction in the total propagation time, as only "half" of the scattering process needs to be simulated. In order to further increase the efficiency of the calculations, we have used

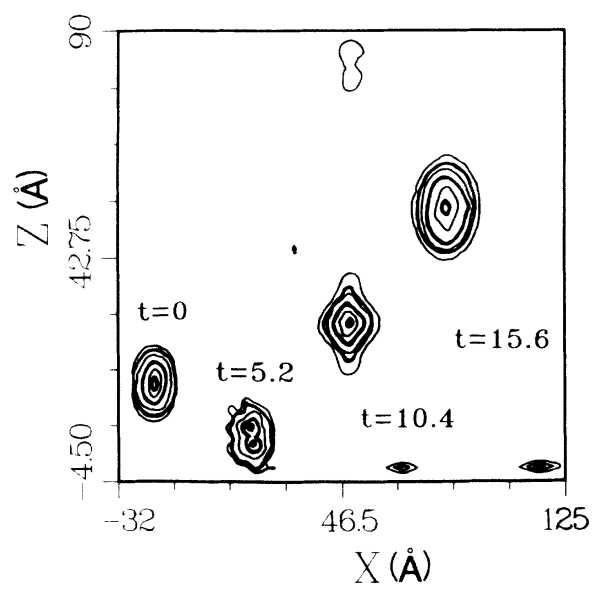

FIG. 4. Contour plot of the probability densities for a sequence of times in the case of the scattering process. The times are in picoseconds and $X$ and $Z$ in angstroms. 
TABLE II. Lifetime $(\tau)$ obtained in the scattering case. $\Gamma(T D)$ computed in this work as the inverse of the lifetime. $\Gamma_{\text {int }}(C C)$ and $\Gamma_{E}(C C)$ resonance widths obtained through $C C$ calculations. Resonance positions $\left(\bar{E}, \bar{\theta}\right.$, and $\left.\varepsilon_{\alpha}\right)$ are also indicated.

\begin{tabular}{|c|c|c|c|c|c|c|c|}
\hline $\begin{array}{l}\text { System and } \\
\text { resonance }\end{array}$ & $\begin{array}{c}\bar{E} \\
(\mathrm{meV})\end{array}$ & $\begin{array}{c}\bar{\theta} \\
(\mathrm{deg})\end{array}$ & $\begin{array}{c}\varepsilon_{\alpha} \\
(\mathrm{meV})\end{array}$ & $\begin{array}{c}\tau \\
(\mathrm{ps})\end{array}$ & $\begin{array}{c}\Gamma(T D) \\
(\mathrm{meV})\end{array}$ & $\begin{array}{c}\Gamma_{\text {int }}(C C) \\
(\mathrm{meV})\end{array}$ & $\begin{array}{c}\Gamma_{E}(C C) \\
(\mathrm{meV})\end{array}$ \\
\hline \multicolumn{8}{|l|}{$\mathrm{He} / \mathrm{Cu}(110)$} \\
\hline$\left[\begin{array}{cc}1 & 0 \\
0\end{array}\right)$ & 13.0 & 54.899 & -4.733 & 142.90 & 0.0046 & 0.0047 & 0.1077 \\
\hline
\end{tabular}

periodic boundary conditions on the grid along the surface. This greatly reduces the number of points needed to carry out an accurate calculation. However, this procedure does have a shortcoming, namely, the limitation in the choice of the parallel component of the wave vector

$$
K_{x, \mathrm{res}}=\frac{\sqrt{2 \mu \bar{E}}}{\hbar}+\frac{2 \pi m}{a},
$$

which must be a multiple of $2 \pi / a$. This condition, together with the resonant condition [Eq. (4)], leads to only a discrete number of incident energies and angles satisfying both equations. Nevertheless, for purposes of comparison with time-independent results, this is not a problem because we can carry out TICC calculations at the desired incidence condition.

From such simulations we have computed lifetimes for the systems $\mathrm{He} / \mathrm{Cu}(110)$ and $\mathrm{He} / \mathrm{Cu}(117)$ at several incidence conditions and resonances. In Table III, grid parameters used in each numerical propagation are shown, labeled by the mean incidence energy. It should be noticed that, although some of the resonances studied are characterized by the same $(m, n, \alpha)$ numbers, they correspond to different incident energies and angles. During the propagation the "restricted norm" [Eq. (8)] was calculated. Figure 5 shows its behavior for a particular case, namely, a mean incidence energy $\bar{E}=28.09 \mathrm{meV}$ for the system $\mathrm{He} / \mathrm{Cu}(117)$. It can be seen that $P_{\mathcal{R}}(t)$ begins with a value of one, indicating that the whole wave packet is inside the region $\mathcal{R}$. (Compare with Fig. 3, where initially the wave packet was far away from the surface.) After a certain period of time the probability density contained into this region starts to decrease. This decay can be accurately fit to an exponential function, from which a lifetime can be estimated. The dynamics of the process is further illustrated in Fig. 6, where snapshots of the probability density of the prepared quasibound state [for the same case of $\bar{E}=28.09 \mathrm{meV}$ and $\mathrm{He} / \mathrm{Cu}(117)]$ are

TABLE III. Grid parameters used in the simulation of the desorption process. Each of the cases studied is indicated by the energy $E$ of the initial wave packet.

\begin{tabular}{lcccc}
\hline \hline$E(\mathrm{meV})$ & 9.582 & 20.719 & 22.877 & 28.087 \\
$n x$ & 128 & 128 & 256 & 256 \\
$n z$ & 512 & 512 & 1024 & 1024 \\
$d x(\AA)$ & 0.08 & 0.08 & 0.07 & 0.07 \\
$d z(\AA)$ & 0.10 & 0.10 & 0.09 & 0.09 \\
\hline \hline
\end{tabular}

presented at different times. All the results are gathered in Table IV, where a comparison with TICC results is made, in the same manner as in the scattering case. As can be seen, the width obtained in this way is in complete agreement with the so-called internal width, corroborating the previous result obtained for the full collision.

\section{DISCUSSION}

We have carried out wave-packet calculations in resonant conditions for the elastic scattering and selective desorption of $\mathrm{He}$ from the $\mathrm{Cu}(110)$ and $\mathrm{Cu}(117)$ surfaces. The main conclusion is that lifetimes in SAR's are directly related to what we have been calling the internal width $\Gamma_{\text {int }}{ }^{4}$ In contrast, the external energy width $\Gamma_{E}$ (or angular width $\Gamma_{\theta}$ ) is the quantity directly obtained from diffraction intensity profiles in an energy (or angular) resolved experiment. Knowledge of the external width provides a way of obtaining the internal width by using Eq. (5) (or the angular version of this equation), as has been already done in several experimental studies. ${ }^{17,18}$ Thus atom-surface resonant scattering constitutes one example where the inverse of the width observed in an energy resolved experiment does not correspond to the lifetime of the resonance. In fact, this would lead to a gross error, since $\Gamma_{E}$ is usually several orders of magnitude larger than $\Gamma_{\text {int }}$. For instance, compare $\tau=142.90 \mathrm{ps,} \mathrm{ob-}$ tained in Table II, versus the value 6.11 ps that would be obtained by using the inverse of the external width. It also follows from the preceding statement that for certain theoretical treatments, care should be taken in the use of total energy $E$ as the independent variable. For instance, in using Smith's lifetime matrix ${ }^{19} Q$, the energy derivative of the scattering matrix should be performed with

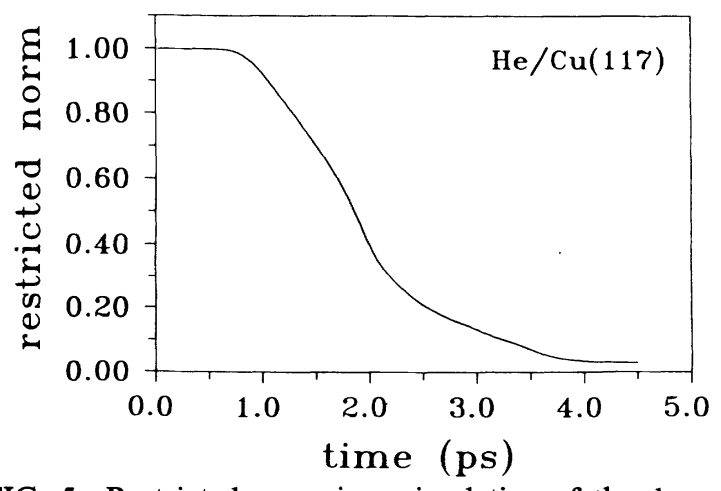

FIG. 5. Restricted norm in a simulation of the desorption process. 

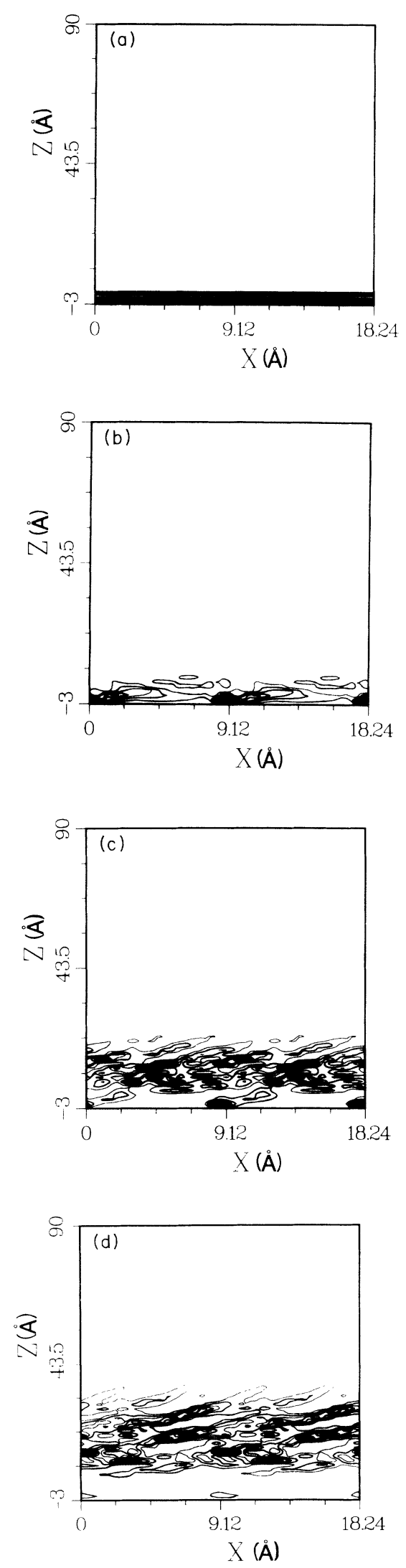

FIG. 6. Contour plot of the probability densities for the simulation of the desorption process at times (a) $t=0.0 \mathrm{ps,} \mathrm{(b)}$ $t=1.5 \mathrm{ps}$, (c) $t=3.0 \mathrm{ps,} \mathrm{and} \mathrm{(d)} t=4.5 \mathrm{ps}$. Axes $X$ and $Z$ in angstroms. respect to the kinetic energy $\mathscr{E}_{\rho}$ instead of the total energy, $E$. Otherwise, results obtained through the $Q$ matrix would not correspond to lifetimes of metastable states. We believe that it is important to appreciate this peculiarity in order to understand the mechanisms of more complicated processes, e.g., inelastic trapping of molecules on surfaces via interaction with phonons.

These wave-packet simulations also provide insight into the qualitative features of the scattering. For instance, in Fig. 4, it can be seen that the wave packet splits after reaching the surface, leaving an "adsorbed" wave packet portion traveling parallel to the surface. By a simple inspection of this figure it can be determined that the speed of the adsorbed wave packet along the surface is 1.3 times the parallel speed of the reflected one. Making use of the simple kinematic relationship that the diffracted wave into the resonant channel should move with a wave vector $K_{x \text {, res }}=K_{x}+2 \pi / a$, for this particular case (with $a=3.6 \AA$ ) we obtain a ratio $K_{x \text {, res }} / K_{x}=1.4$. Thus the wave-packet simulation confirms the traditional qualitative interpretation of selective adsorption resonances in a nice way. ${ }^{13}$

The simulation of the selective desorption process provides very good approximations to the resonance lifetimes. The initial wave packet, constructed on the basis of a diabatic separation, has proved to be nearly a single resonance state. As can easily be appreciated, if the initial wave packet contained a considerable portion of nonresonant state, the fitting should be done at longer times in order to give the nonresonant part of the wave packet a chance to escape before computation of $P_{\mathscr{R}}(t)$ is attempted. This, however, has not been the case in the systems considered here, since the fitting can be carried out immediately after the first portion of wave packet begins to depart from the interaction region. A further test to check this assertion and on the accuracy of the procedure was carried out through an application of Prony's method. This method, which involves fitting the autocorrelation function to a sum of complex exponentials, ${ }^{12(a)}$ is powerful enough to enable extraction of resonance lifetime data from an initial state which consists of a superposition of (several) resonance states as well as continuum contributions. We should note that for a well-localized wave packet (instead of our extended one) the autocorrelation function would be nonsensical. This analysis led to the same width previously obtained by the simpler fitting to only one exponential function, and furthermore, it gave a percentage of about $96 \%$ of the initial wave packet as the quasibound state responsible for that width. This could serve as a quantitative measure of the accuracy of the diabatic separation. Again we stress that even if the diabatic separation had not worked very well, we still would be able to obtain good values for lifetimes via Prony's method. ${ }^{12(a)}$ On the basis of all the points stressed above, we can conclude that the time-dependent wave packet approach is very promising for getting good estimations of lifetimes, even more so in the presence of defects in the surface, where close-coupling-type schemes cannot be applied. ${ }^{8(a)}$

Concerning the efficiency of the calculations carried out, the use of periodic boundary conditions proved to be 
TABLE IV. Same as in Table II but for the case of the half-collision process and for different systems and resonances as indicated.

\begin{tabular}{|c|c|c|c|c|c|c|c|}
\hline $\begin{array}{l}\text { System and } \\
\text { resonance }\end{array}$ & $\begin{array}{c}\bar{E} \\
(\mathrm{meV})\end{array}$ & $\begin{array}{c}\bar{\theta} \\
(\mathrm{deg})\end{array}$ & $\begin{array}{c}\varepsilon_{\alpha} \\
(\mathrm{meV})\end{array}$ & $\begin{array}{c}\tau \\
\text { (ps) }\end{array}$ & $\begin{array}{l}\Gamma(T D) \\
(\mathrm{meV})\end{array}$ & $\begin{array}{c}\Gamma_{\text {int }}(C C) \\
(\mathrm{meV})\end{array}$ & $\begin{array}{c}\Gamma_{E}(C C) \\
(\mathrm{meV}) \\
\end{array}$ \\
\hline $\mathrm{He} / \mathrm{Cu}(110)$ & 9.582 & 54.582 & -4.737 & 103.82 & 0.0063 & 0.0063 & 1.261 \\
\hline$\left(\begin{array}{cc}1 & 0 \\
1\end{array}\right]$ & 20.719 & 56.234 & -4.736 & 253.16 & 0.0026 & 0.0023 & 0.033 \\
\hline $\mathrm{He} / \mathrm{Cu}(117)$ & 22.877 & 31.36 & -1.95 & 0.878 & 0.75 & 0.73 & 1.61 \\
\hline$\left(\begin{array}{cc}5 & 0 \\
1\end{array}\right)$ & 28.087 & 34.31 & -1.91 & 1.02 & 0.62 & 0.62 & 1.50 \\
\hline
\end{tabular}

very convenient because of the reduction of grid points needed to perform an accurate propagation, resulting in a large saving of computational time. However, as mentioned above, it has the disadvantage that they can be only used for a specific set of energies, subject to the requirements of Eqs. (4) and (12). As a matter of fact, in our case, from previous TICC calculations we know that the set of energies used in the simulation of the selective desorption corresponded to incidence conditions where several resonances were very close. This prevented us from performing the scattering calculation presented in Sec. III A for the same resonances as were used in the simulation of the desorption process presented in Sec. III B. In a general case, when simulating the desorption process, the method of approximating a plane wave by a sum of Gaussian functions (as was done in the case of the scattering calculations reported here) would have to be used. This will probably increase the number of grid points needed, but it allows us to work at the desired energy and incidence angle. The method of Gaussian superposition is very useful, since by adding more Gaussian functions over a more extended region the wave packet becomes more "monoenergetic," avoiding (although not completely) effects of overlapping resonances. On the other hand, demanding a smaller spread in energy implies a greater numerical effort, because a more extended wave packet requires a larger spatial grid as well as a longer propagation time. This means that in practice there is a limit beyond which it is very difficult to reach sharper energy resolution.

Another way of increasing the efficiency of the full collision calculation lies in the choice of Gaussian parameters in Eq. (9). They were determined from a "backward" semiclassical propagation ${ }^{11}$ starting with a well-focused Gaussian wave packet in the interaction region. Then, during the quantal propagation, the wave packet is able to "shrink" as it approaches the surface, thus minimizing its spatial spread as it is being scattered back, and consequently allowing the use of a smaller grid.

For the simulation of both desorption and scattering processes, the use of absorbing boundary conditions ${ }^{20}$ could greatly reduce the number of grid points along the perpendicular direction to the surface, although we have not used them here. Since we calculate the restricted norm integrating over a small part of the space, the absorption of the wave packet into the grid boundary would not affect the results. The reduction in grid size would enable longer simulation of the adsorption/desorption process and hence a more accurate determination of resonance lifetimes.

Regarding the method of lifetime extraction utilized here, i.e., fitting the restricted norm to an exponential function, an alternative treatment might entail calculation of the "time delay" undergone by the wave packet as compared with a free propagation. ${ }^{21}$ Some work in this direction is now in progress.

Finally, the small discrepancy between widths computed by time-dependent and TICC methods merits some comments. It should be mentioned that although TICC is termed an "exact" method (provided that a sufficient number of channels has been included to ensure numerical convergence), the way in which the widths are computed through the exact $\mathcal{S}$ matrix necessitates some kind of fitting of the diffraction intensities to a Lorentzian function or through the so-called $\mathcal{S}$-mixed matrix method. ${ }^{22}$ The result obtained via the time-dependent fitting could be more accurate. First, fitting to an exponential function might well be subject to a smaller error than fitting to a Lorentzian function. Second, and more important, the use of the scattering matrix for studying resonances, within the isolated narrow resonance assumption, assumes knowledge of the so-called "background scattering matrix," 10 which corresponds to the scattering process in the absence of resonances. In general this matrix is unknown, and therefore a model needs to be adopted or some behavior assumed (a common assumption relies on extrapolation of off-resonance $\mathcal{S}$-matrix values into the resonance region). Often the actual behavior is not so trivial. For instance, in the case of very corrugated surfaces such as $\mathrm{He} / \mathrm{Cu}(117)$, the base line of diffraction intensities by no means follows a smooth trend. Therefore, the errors committed in methods that make use of the scattering matrix (such as the $\mathcal{S}$-mixed matrix or the lifetime matrix $Q$ ) due to assumptions about the behavior of the background scattering matrix may be larger than errors that could arise in procedures similar to the one employed here.

Taking into account all the issues raised in the preceding paragraphs, time-dependent wave-packet propagation methods for studying the behavior of resonance states may be competitive even from the computational point of view. 


\section{CONCLUDING REMARKS}

In the cases presented here the distinction between external and internal widths leads to important differences in the evaluation of adsorption lifetimes. How this would affect more complicated situations where phonons or defects are involved is a difficult but important question. These effects are very difficult to evaluate without the benefit of extensive calculations. However, some general ideas can be outlined. Within the TICC framework, treatment of inelastic effects (phonons) translates into the addition of many extra diagonal and off-diagonal terms in Eq. (2), i.e., the inclusion of many more channels, the inelastic diffraction channels. Bearing this in mind, the nonlinear relation between total energy and kinetic energy will persist, although there will be some extra factors involving the phonon wave vector and the crystal energy. Therefore the distinction between internal and external widths will hold and the relationship between them will be given by an equation analogous to Eq. (5). Moreover, interesting effects that arise from the analysis of the relationship between the internal and the external width (such as the critical kinematic effect ${ }^{4}$ ) could certainly have a counterpart in inelastic scattering. ${ }^{23}$

In this context, it becomes apparent that further work in this direction is needed and that wave packet methods may be useful in improving our understanding of these processes. Indeed, wave packet techniques have been already successfully applied to the study of scattering and photodesorption of atoms and diatomic molecules from surfaces at finite temperatures, ${ }^{24}$ as well as in the case of nonperiodic surfaces [see Refs. 7 and 8(a) for cross references on this topic]. However, as far as we know, computations including phonons in resonant cases have not been reported yet using these formalisms. In order to get a qualitative idea of what could happen, it may suffice to consider time-dependent Hartree (TDH) level coupling of the interacting molecule to the phonons, as was done in Ref. 24. In the case of surfaces with imperfections or defects, the use of wave-packet methodologies seems even more natural since the lack of periodicity prevents the use of the usual TICC expansion. Averaged results obtained from distributing the defects statistically in an appropriate manner could adequately emulate moleculesurface interactions on nonperiodic surfaces. However, the long time propagation required in a resonant case may compromise the accuracy of the TDH approach. In such cases it may be possible to incorporate corrections to the TDH level dynamics ${ }^{25}$ without undue effort.

\section{ACKNOWLEDGMENTS}

We are grateful to S. K. Gray for providing us with his code on "Prony's method." M.I.H. gratefully acknowledges a grant from the Comunidad de Madrid in Spain and financial support from the same institution during her stay at the University of Pittsburgh. This work has been partially supported by the CICYT Grant No. PB87-0272 and Comunidad de Madrid Grant No. 064/92. R.D.C. wishes to acknowledge National Science Foundation Grant No. CHE-9101432 for partial financial support, and the Pittsburgh Supercomputer Center for a grant of computer time.
${ }^{1}$ (a) Helium Atom Scattering from Surfaces, edited by E. Hulpke, Springer Series in Surfaces Sciences Vol. 27 (Springer-Verlag, Berlin, 1992); (b) J. Lapujoulade, in Interaction of Atoms and Molecules with Solid Surfaces, edited by V. Bortolani, N. H. March, and M. P. Tosi (Plenum, New York, 1990); (c) K. Kern and G. Compsa, Adv. Chem. Phys. 76, 211 (1989); (d) J. P. Toennies, in Dynamics of Gas-Surface Interaction, edited by G. Benedek and U. Valbusa (Springer-Verlag, Berlin, 1982); (e) T. Engel and K. H. Rieder, in Structural Studies of Surfaces with Atomic and Molecular Beam Diffraction (Springer-Verlag, Berlin, 1982); (f) H. Hoinkes, Rev. Mod. Phys. 52, 933 (1980); (g) N. Cabrera, V. Celli, F. O. Goodman, and J. R. Manson, Surf. Sci. 19, 67 (1970).

${ }^{2}$ H. Hoinkes and H. Wilsch, in Helium Atom Scattering from Surfaces [Ref. 1(a)], and references therein.

${ }^{3}$ S. Andersson, L. Wilzén, M. Persson, and S. Harris, Phys. Rev. B 40, 8146 (1989); Böheim, Surf. Sci. 148, 463 (1984).

${ }^{4}$ M. Hernández, S. Miret-Artés, P. Villarreal, and G. DelgadoBarrio, Surf. Sci. 251/252, 369 (1991); M. Hernández, S. Miret-Artés, P. Villarreal, and G. Delgado-Barrio, ibid. 274, 21 (1992); S. Miret-Artés, M. Hernández, J. CamposMartínez, P. Villarreal, and G. Delgado-Barrio, in Trends in Applied Theoretical Chemistry, edited by L. A. Montero and Y. G. Smeyers (Kluwer Academic, Dordrecht, 1992).

${ }^{5}$ D. Kosloff and R. Kosloff, J. Comput. Phys. 52, 35 (1983); R. Kosloff and D. Kosloff, J. Chem. Phys. 79, 1823 (1983).

${ }^{6}$ A. T. Yinnon and R. Kosloff, Chem. Phys. Lett. 102, 216
(1983).

${ }^{7}$ R. B. Gerber, R. Kosloff, and M. Berman, Comput. Phys. Rep. 5, 61 (1986); R. B. Gerber, Chem. Rev. 87, 29 (1987); G. D. Billing, Comput. Phys. Rep. 12, 383 (1990), and references therein.

${ }^{8}$ (a) A. T. Yinnon, R. Kosloff, and R. B. Gerber, J. Chem. Phys. 88, 7209 (1988); (b) R. Kosloff and C. Cerjan, ibid. 81, 3722 (1984).

${ }^{9}$ R. C. Mowrey and D. J. Kouri, J. Chem. Phys. 86, 6140 (1987); 84, 6466 (1986), and references therein.

${ }^{10}$ J. R. Taylor, Scattering Theory (Wiley, New York, 1972), p. 411.

${ }^{11}$ Drolshagen and Heller, J. Chem. Phys. 79, 2072 (1983); G. Drolshagen and R. Vollmer, Chem. Phys. Lett. 122, 333 (1985).

${ }^{12}$ (a) S. K. Gray, J. Chem. Phys. 96, 6543 (1992); (b) Chem. Phys. Lett. 197, 86 (1992); (c) S. K. Gray and C. E. Wozny, J. Chem. Phys. 91, 7671 (1989).

${ }^{13}$ J. E. Lennard-Jones and A. F. Devonshire, Nature 137, 1069 (1936); J. E. Lennard-Jones and A. F. Devonshire, Proc. R. Soc. London Ser. A 158, 253 (1937).

${ }^{14} \mathrm{M}$. Hernández, O. Roncero, S. Miret-Artés, P. Villarreal, and G. Delgado-Barrio, J. Chem. Phys. 90, 3823 (1989).

${ }^{15}$ D. Gorse, B. Salanon, F. Fabre, A. Kara, J. Perreau, G. Armand, and J. Lapujoulade, Surf. Sci. 147, 647 (1984).

${ }^{16}$ M. D. Feit, J. A. Fleck, Jr., and A. Steiger, J. Comput. Phys. 47, 412 (1982). 
${ }^{17}$ G. Brusdeylins, R. B. Doak, and J. P. Toennies, J. Chem. Phys. 75, 1784 (1981).

${ }^{18}$ J. Lapujoulade, J. Perreau, and A. Kara, Surf. Sci. 129, 59 (1983).

${ }^{19}$ F. T. Smith, Phys. Rev. 118, 349 (1960).

${ }^{20}$ R. Kosloff and D. Kosloff, J. Comput. Phys. 63, 363 (1986); D. Neuhauser, M. Baer, R. S. Judson, and D. J. Kouri, Comput. Phys. Commun. 63, 460 (1991).

${ }^{21}$ A. Böhm, Quantum Mechanics: Foundations and Applications (Springer-Verlag, Berlin, 1986).
${ }^{22}$ G. Delgado-Barrio, P. Villarreal, P. Mareca, and J. A. Beswick, Int. J. Quantum Chem. 27, 173 (1985).

${ }^{23}$ S. Miret-Artés and G. Benedek (unpublished).

${ }^{24}$ M. Messina and R. D. Coalson, J. Chem. Phys. 95, 5364 (1991); 95, 8977 (1991); B. Jackson, ibid. 92, 1458 (1990); A. J. Cruz and B. Jackson, ibid. 91, 4985 (1989); B. Jackson and H. Metiu, ibid. 83, 1952 (1985); 85, 4129 (1986); B. Jackson, ibid. 89, 2473 (1988); 90, 140 (1989).

${ }^{25}$ J. Campos-Martínez and R. D. Coalson, J. Chem. Phys. 93, 4740 (1990); 99, 9629 (1993). 